\title{
ÁCAROS PARÁSITOS EN PESTAÑAS Y PÁRPADOS HUMANOS. ANÁLISIS DE PREVALENCIA DE DEMODEX FOLLICULORUM EN CONSULTAS OFTALMOLÓGICAS EN LA CIUDAD DE CÓRDOBA, ARGENTINA.
}

Pizzi, Hugo L.*2

Pizzi, Rogelio D. ${ }^{* * 2}$ Maffrand, Carola M. ${ }^{* \star \star \star}$ Tomas, Axel ${ }^{\star * * 2}$

*Profesor Titular Cátedra de Clínica Oftalmológica. **Profesor Titular Plenario Cátedra de Parasitología y Micología Médicas.

${ }^{* * *}$ Profesores Cátedra de Parasitología y Micología Médicas.

***Médica oftalmóloga, FCM. UNC.

${ }^{1}$ Roque Alejandro Maffrand. Belgrano 511. Córdoba, Argentina.roquemaffrand@ gmail.com

2Departamento Publicaciones del Centro de Enfermedades Tropicales y Cátedra de Parasitología y Micología Médicas. Facultad de Ciencias Médicas.

Universidad Nacional de Córdoba., Argentina.

Trabajo recibido: 23 de Agosto de 2016.

Aprobado: 13 de Marzo de 2017.
PARASITIC MITES IN HUMAN EYELASHES AND EYELIDS. PREVALENCE ANALYSIS OF DEMODEX FOLLICULORUM IN OPHTHALMOLOGICAL CONSULTATIONS IN THE CITY OF CORDOBA, ARGENTINA.

ÁCAROS PARASITAS EM CÍLIOS E PÁLPEBRAS HUMANAS. ANÁLISE DA PREVALÊNCIA DO DEMODEX FOLLICULORUM EM CONSULTAS OFTALMOLÓGICAS, NA CIDADE DE CÓRDOBA, ARGENTINA.

\section{Resumen}

El presente es un estudio de prevalencia sobre la infestación por Demodex folliculorum, ácaro parásito de las pestañas, folículos y de los conductos de las glándulas de meibomio. Es de observación frecuente este tipo de parásitos en la consulta oftalmológica. Los pacientes presentan blefaritis con prurito, ardor, irritación y dolor ocular, reciben rápido alivio luego del diagnóstico y el tratamiento acaricida.1,4,14, 30, 31 Este informe investigó la totalidad de pacientes que concurrieron a la consulta oftalmológica en la Cátedra de Oftalmología del 
Hospital Nacional de Clínicas, Facultad de Ciencias Médicas de la Universidad Nacional de Córdoba, Argentina. El objetivo fue determinar la prevalencia del ácaro en las consultas oftalmológicas con diagnóstico de blefaritis y en segundo término que tipo de síntomas y signos acompañan la afección. El número total de pacientes que consultaron en los 243 días hábiles del año 2012, fue de $48.600(n=48.600)$. En el $50 \%$ de los pacientes, $(n=24.300)$, se diagnosticó blefaritis; en el $30 \%(n=7.290)$, de éstos, la blefaritis se debió a la presencia del ácaro. De lo dicho se concluye que, el $15 \%$ de los pacientes que consultaron lo hicieron por blefaritis por Demodex folliculorum. La infestación por el ácaro siempre se asocia en nuestra experiencia con blefaritis. Otros autores, 7,16,19 señalan el hallazgo del parásito sin la presencia de síntomas. La blefaritis se define como la inflamación de los párpados y pudimos determinar que el $100 \%$ de estos pacientes presentó prurito ocular, el $60 \%$ sensación de ojo seco, el $50 \%$, lagrimeo, el $40 \%$ ardor, el $30 \%$,dolor ocular, el $30 \%$, secreciones y el $20 \%$ visión borrosa. La afección se torna crónica y lleva en ocasiones a la pérdida de las pestañas. En la mayoría de los casos los pacientes conviven con el cuadro clínico y retrasan la consulta. Resulta sorprendente ver varios individuos de esta especie de ácaros moviéndose entre las pestañas. El estudio expone la alta prevalencia de la blefaritis acaricida teniendo en cuenta una muestra importante de consultas oftalmológicas y tiene el propósito de contextualizar estadísticamente la afección y poner al corriente sobre estos resultados a los profesionales médicos. En último término, la blefaritis por Demodex folliculorum es una patología subestimada que debe ser oportunamente reconocida en la práctica médica general.

Palabras Clave: Ácaros del hombre, Demodex folliculorum y blefaritis.

\section{Abstract}

This is a prevalence study on Demodex folliculorum infection, a parasitic mite of human eyelashes, eyelids and meibomian glands ducts. This type of mites frequently appears in opthalmologic consultations. Patients present blepharitis with pruritus, burning sensation, irritation and eye pain, which are quickly relieved after diagnosis and mite treatment.1,4,14, 30, 31 All the patients who visited the ophthalmology office in the Chair of Ophthalmology at Hospital Nacional de Clinicas, School of Medicine, National University of Cordoba, Argentina, were included in this research. The objective was to determine the presence of this mite in opthalmologic consultation when blepharitis was diagnosed, and secondly, define what type of symptoms and signs appear with this condition. The total number of patients seen in the 243 working days of the year 2012 were 48,600 $(n=48,600)$. Half of these patients $(n=24,300)$ were diagnosed with blepharitis; in $30 \%$ of the cases $(n=7230)$, blepharitis was due to the mite. From this, we conclude that $15 \%$ of the patients consulted because of blepharitis caused by Demodex folliculorum. In our experience mite infection is always associated with blepharitis. Other authors, 7,16,19 have pointed to the presence of mites without symptoms. Blepharitis is defined as eyelid inflammation and we could determine that $100 \%$ of these patients presented eye pruritus, $60 \%$ dry eye sensation, $50 \%$ tearing, $40 \%$ burning, $30 \%$ eye pain, $30 \%$ discharges and 20 $\%$ blurred vision. This condition becomes chronic and in occasions it may cause loss of eyelashes. In most cases, patients take a long time before seeing a doctor because of this condition. It is surprising to see this kind of mites moving around eyelashes. This study exposes the high prevalence of mite blepharitis taking into account an important sample of opthalmologic consultations and tries to statistically contextualize the condition and inform medical professionals about these results. Finally, blepharitis due to Demodex folliculorum is an underestimated pathology which should be appropriately recognized in general medical practice.

Key Words: Human mites, Demodex folliculorum and blepharitis. 
Este é um estudo de prevalência na infestação do Demodex folliculorum, ácaro parasita dos cílios, folículos e ductos das glândulas meibomianas. É frequente a observação deste tipo de parasitas na consulta oftalmológica. Os pacientes que apresentam blefarite com coceira, ardor, irritação e dor nos olhos, recebem alívio imediato após o diagnóstico e tratamento acaricida.1,4,14, 30, 31 Este relatório pesquisou todos os pacientes que frequentam a consulta oftalmológica na Cadeira de Oftalmologia do Hospital Nacional das Clínicas da Faculdade de Ciências Médicas da Universidade Nacional de Córdoba, Argentina. $\mathrm{O}$ intuito foi determinar a prevalência do ácaro em consultas oftalmológicas com diagnóstico de blefarite e em segundo lugar que tipo de sinais e sintomas acompanham a doença. O número total de pacientes consulentes nos 243 dias úteis em 2012 era 48.600 $(\mathrm{n}=48.600)$. Em 50\% dos pacientes $(\mathrm{n}=24300)$, diagnosticou-se blefarite; em $30 \%(\mathrm{n}=$ 7290 ) destes, a blefarite ocorreu pela presença do ácaro. Do anteriormente dito concluise que $15 \%$ dos pacientes consultaram por causa da blefarite por Demodex folliculorum. Em nossa experiência, a infestação deste ácaro é sempre associada com blefarite. Outros autores, 7,16,19 reconhecem a presença do parasita com a ausência de sintomas. Blefarite é definida como uma inflamação das pálpebras e conseguimos determinar que $100 \%$ destes pacientes mostrou prurido ocular, $60 \%$ sensação de olho seco, $50 \%$, lacrimação, $40 \%$ ardor, $30 \%$, dor ocular, $30 \%$ secreções e $20 \%$ visão turva. A condição vira crônica e às vezes leva à perda dos cílios. Na maioria dos casos, os pacientes convivem com sintomas clínicos e adiam a consulta. É surpreendente ver vários indivíduos dessa espécie de ácaros que se deslocam entre os cílios. O estudo expõe a alta prevalência da blefarite acaricida analisando uma amostra importante de consultas oftalmológicas e visa contextualizar a doença estatisticamente, colocando estes resultados ao dispor dos profissionais médicos. Em última análise, blefarite por Demodex folliculorum é uma patologia subestimada que deve ser oportunamente reconhecida na prática médica geral.

Palavras-chave: Ácaros do homem, Demodex folliculorum e blefarite.

\section{Introducción}

Demodex spp.(griego: demos = grasa; $d$ ex= carcoma, insecto roedor de madera), es un artrópodo arácnido quelicerado perteneciente a la clase arachnida, del orden acarina, suborden trombidiforme. El parásito fue descubierto por Berger en 1841, en el conducto auditivo externo humano, en el mismo año Henle, lo encuentra en un paciente con acné. Owen (1843), propuso el término Demodex folliculorumy Becker, en 1875 lo observó en el ducto de una glándula de meibomio y Majocchi en 1878,lo ubica en un chalazión de un paciente con blefaritis crónica. En 1963, Akbulotova determina dos subespecies que afectan al hombre, el Demodex folliculorum longus y Demodex folliculorum brevis. Demodexspp.es un parásito vermiforme de $0,1 \times 0,4 \mathrm{~mm}$ de amplia distribución en el mundo1, 4, 18, 21, 22.(fig. 1). Subsiste cierta controversia en nuestro tiempo acerca de su rol patógeno en la producción de enfermedad en el hombre.16,19 Demodex folliculorum presenta una localización a nivel de los folículos pilosos de las pestañas, glándulas de meibomio y es productor de blefaritis en el hombre; Demodex brevis se radica en la piel de nariz, mejillas, surco nasogeniano, cuello, cuero cabelludo y su rol patógeno presenta cierta controversia.1,18,19,21,22 Demodexspp. contiene un gnatosoma, llamado también capítulo o cabeza pequeña, bien desarrollado con las piezas bucales directamente implantadas en el cuerpo, las que incluyen al hipostoma, los quelíceros y los pedipalpos. El cuerpo carece de una nítida diferenciación en cabeza, tórax y abdomen, pero se considera que está formado por metámeros fusionados entre sí. El idiosoma corresponde al verdadero cuerpo de los acarinos que puede estar dividido o no en diferentes regiones, las que están señaladas por la inserción de las patas; éstas están formadas por coxa, trocánter, fémur, 
tibia y tarso. En el adulto hembra hay un tocostoma o apertura genital para la postura de los huevos en el idiosoma. Son de sexos separados, de metamorfosis incompleta o simple con huevos, ninfas hexápodas y adultos octópodos.1, 4,18 Esta especie de ácaro carece de aparato respiratorio, tiene cuerpo muy alargado, coniforme, con forma de gusano y es de color gris claro. El sistema excretor está compuesto por los tubos de Malpighi, en número, forma y disposición variados y las glándulas coxales. En la piel, presentan interés médico, Demodex folicullorum y Demodex brevis, éstos destruyen células epiteliales, sebáceas, se alimentan del sebo humano y son parásitos del hombre. 1,18,21,22, 27

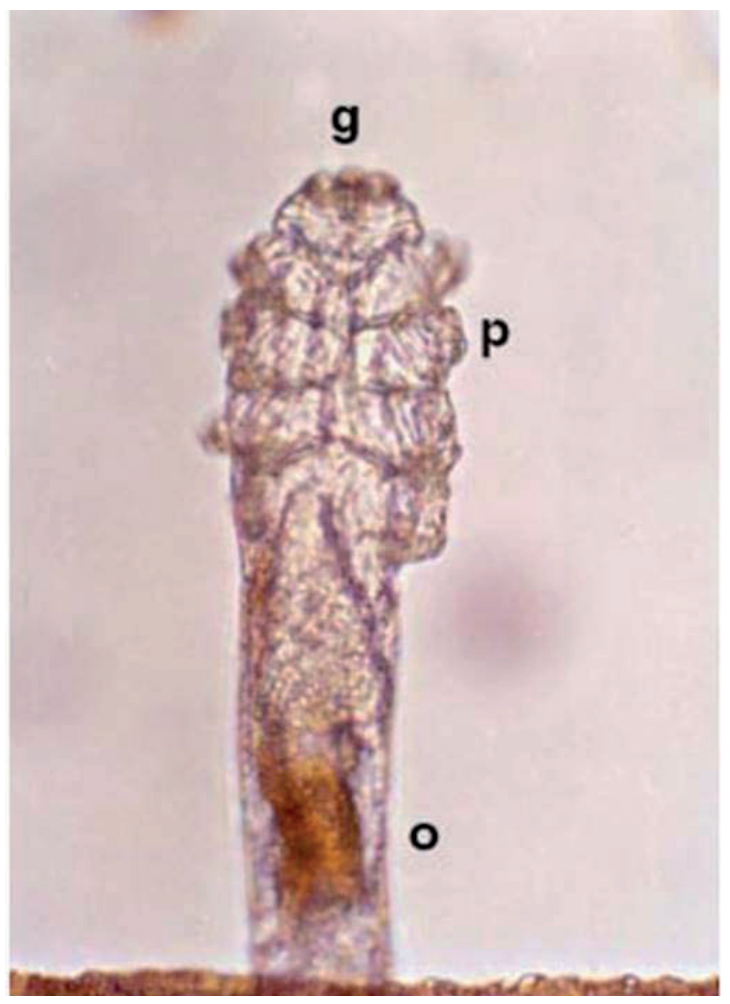

Figura $\mathrm{N}^{\mathrm{o}} 1$ : Microfotografía de Demodex folliculorum.

Vista ventral; g: gnatosoma con 4 pares de patas y o: opistosoma, abdomen. Aumento 500x Corredor-Osorio. UNAM

\section{Ciclo biológico}

La hembra luego de la cópula, se abre camino dentro de la glándula sebácea para depositar sus huevos, en el caso del Demodex folliculorum, en el folículo piloso. Una vez que nacen las larvas se alimentarán continuamente y producirán una muda para dar origen a una protolinfa, (dentro del conducto piloso o sebáceo), luego se diferenciará en una deutoninfa que se dirige a la piel y reingresa al folículo para alcanzar el estado adulto. La hembra esperará al macho en la abertura del folículo para copular.1,4,18, 21, 22

El ciclo completo le demanda al parásito un total aproximado de 14 días. Es interesante destacar que Demodexspp. huye siempre de la luz, por lo que tiene fototaxia negativa. Demodex brevis se localiza en glándulas sebáceas sobre nariz, piel de la cara, conducto auditivo externo e incluso en cuero cabelludo por lo general, en número de un ácaro o a lo sumo dos. En cambio, Demodex folliculorum que habita los ductos pilo sebáceos, se lo encuentra en mayor concentración en los folículos del párpado inferior. Esta localización y sus hábitos gregarios de 3 o más individuos por folículo nos permite comprender la 
fisiopatología de la blefaritis.4, 8, 25, 26 Generalmente se encuentra una sola especie en las localizaciones referidas, aunque en ocasiones se han encontrado las dos especies juntas. $1,4,18,26$

\section{Clínica}

La acción patógena de Demodex spp. está en discusión, 7, 19 pero la parasitación de los folículos de los párpados por D. folliculorum lleva a una blefaritis (Fig. 2) con abundante secreción, eritema, prurito, ardor, dolor, ojo seco, lagrimeo y caída de las pestañas..4,5, 8,19 Demodex spp. se señala como co-productor de un acné demodécico con comedones, asociado a estafilococos piógenos.2,9 Las pieles grasas o con componente seborreico se constituyen como un factor predisponente para la colonización por parte de los ácaros. 2,9,13, $28 \mathrm{La}$ edad actuaría de igual manera, ya que, en adultos y especialmente en ancianos, las tasas de prevalencia son mayores.30Tradicionalmente no se adjudicaba un papel de importancia al Demodex spp. con respecto a la producción de enfermedad en el hombre, hoy se considera que agravaría los cuadros de acné y rosácea pero, más aún, en los pacientes con SIDA puede provocar importantes lesiones papulo-nodulares en cara, cuello y cabeza que motivan la inmediata indicación de tratamiento.2,9,13,19, 27

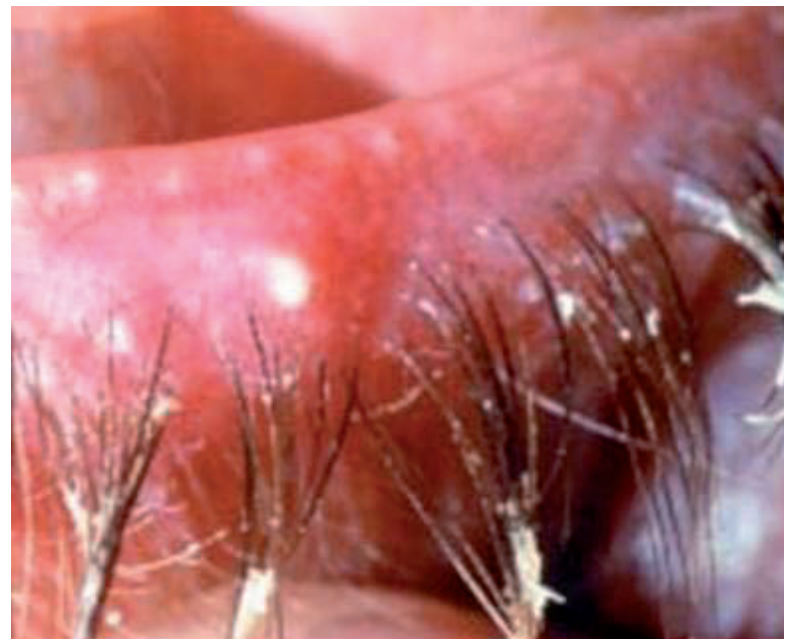

Figura $\mathrm{N}^{\circ} 2$ : Blefaritis por Demodex folliculorum.

Eritema y abundante secreción que pega las pestañas

La colonización de los folículos pilosos de las pestañas genera una importante blefaritis que puede ser más notoria a nivel del párpado inferior con abundante secreción que pega las pestañas en especial luego del sueño nocturno. Esta afección produce la pérdida de las pestañas. Utilizando la lámpara de hendidura se pueden ver los parásitos y residuos sebáceos, escamas e incluso un eritema en la base de las pestañas (Fig.2). Más aún, como también coloniza las glándulas de meibomio, es capaz de producir meibomitis y el orzuelo interno crónico. 2, 4,25,32

Una demodicidosis similar a rosácea que compromete los párpados con eritema, sequedad, descamación fina en la cara se describe en mujeres que utilizan cremas limpiadoras y otros cosméticos sin el empleo de agua y jabón para limpiar la piel. Estudios señalan que Demodex spp. tendría una acción como vector o acarreador de bacterias hacia las profundidades del folículo o las glándulas sebáceas, ya que se las ha identificado en la superficie del cuerpo e incluso en el interior del ácaro. El fondo de los folículos se constituiría en un reservorio importante de bacterias que podrían generar infecciones en estas localizaciones con la correspondiente expresión clínica. 2,9,13, 26, 28,32 
Se trató de un estudio de prevalencia realizado con la totalidad de los pacientes que concurrieron en el marco de las consultas espontáneas en la Cátedra de Oftalmología de la FCM, UNC; Hospital Nacional de Clínicas de la ciudad de Córdoba, Argentina. El estudio se llevó a cabo en los 243 días laborables del año 2012 y la suma total de pacientes examinados fue de $48.600(\mathrm{n}=48.600)$. No se incluyeron niños ni adolescentes. Cada paciente fue examinado y notificado en caso de diagnóstico para blefaritis por Demodex folliculorum y se le indicó el tratamiento farmacológico. El examen oftalmológico se realizó con observación directa de los ojos de cada paciente y mediante lámpara de hendidura y filmación en películas de video. Los elementos a valorar para el diagnóstico clínico de la blefaritis fueron prurito, sensación de ojo seco, dolor ocular, lagrimeo, visión borrosa, ardor, secreciones, escamas en los bordes palpebrales con pérdidas de las pestañas en ocasiones. Mediante la utilización de la lámpara de hendidura se observaron los ácaros en movimiento entre pestañas y bordes palpebrales. Además se realizó microscopía óptica para aplicación de método parasitológico directo consistente en la extracción de 1 (una) pestaña por cada párpado, colocación sobre portaobjetos y se cubrió con cinta adhesiva.

\section{Resultados}

El total de la muestra $(n=48.600)$ de pacientes arrojó un promedio de 200 consultas por día. Se realizó el diagnóstico de blefaritis en 24.300 consultas ( $\mathrm{n}=24300)$, lo cual arrojó un porcentaje del $50 \%$ de los pacientes que concurrieron al examen general de oftalmología. Dentro del grupo de los pacientes diagnosticados con blefaritis, el 30\% $(\mathrm{n}=7290)$ correspondió a blefaritis por ácaros (Demodex folliculorum); el $100 \%$ de éstos presentó prurito, el $60 \%$ ojo seco, lagrimeo el $50 \%$, ardor el $40 \%$, dolor ocular el 30 $\%$, secreciones el $30 \%$ con presencia de escamas y visión borrosa el $20 \%$. El prurito se presenta a distintas horas del día y lleva a irritación ocular.

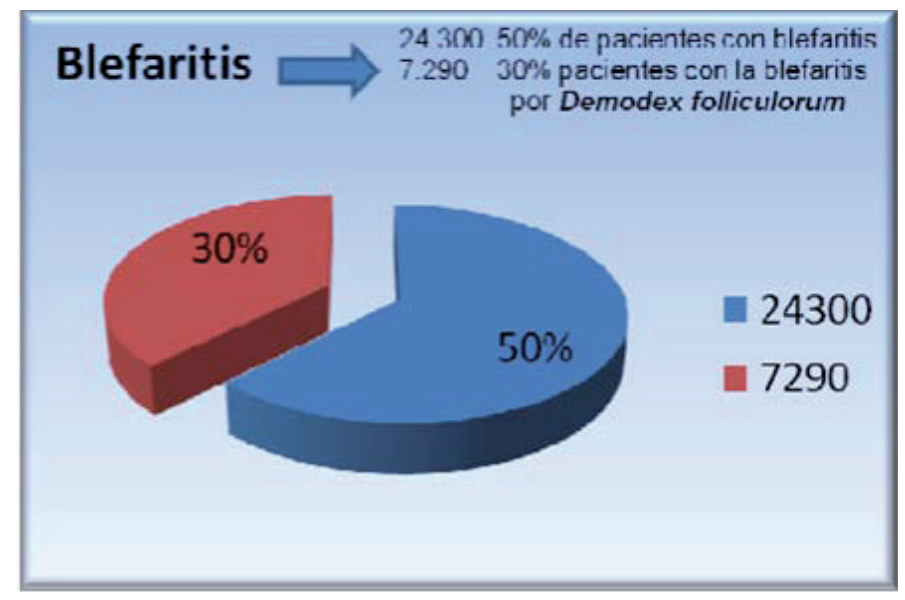

Presencia de blefaritis en la Consulta Oftalmológica.

\section{Discusión y Conclusión}

En compulsa con otros autores 7, 19 quienes sostienen la presencia del parásito sin síntomas, la presencia de la infestación por Demodex folliculorum implica según nuestra observación, el acompañamiento de clínica ocular en todos los casos; por lo que no puede decirse que el ácaro sea solo un saprófito. Otros autores, 16 sostienen que la parasitosis 
no se acompaña de síntomas sólo en un $8 \%$ de los pacientes. La investigación señaló que del total de consultas oftalmológicas, las blefaritis corresponden al $50 \%$. En concordancia con el estudio citado comparativamente, 16 no todos los pacientes con síntomas de blefaritis tienen el ácaro, en un $20 \%$ de los casos no se encontró el parásito. El estudio contrastado 16 fue de prevalencia general y arrojó que el $61 \%$ de los ojos examinados presentaba Demodex folliculorum, en un $39 \%$ de esos pacientes no se encontraba el ácaro y el $66,6 \%$ de estos pacientes tenían síntomas. En nuestro caso, el estudio mostró que el $50 \%$ de las consultas oftalmológicas se corresponden con blefaritis, el $30 \%$ presentó el ácaro y que en este $30 \%$ siempre se encuentran síntomas. Gao YY et al.8informan aspectos controversiales de distintos autores y la relación entre clínica ocular y hallazgos de Demodex follicullorum especificando deficiencias metodológicas en la búsqueda del parásito. En tal sentido señalan que frente a clínica ocular sospechosa de blefaritis acaricida, el hallazgo del parásito tuvo un porcentaje de $100 \%$ cuando se lo buscó en pestañas con evidencia de parasitación consistente en la formación de cilindros de secreción alrededor del eje longitudinal de las mismas. A pesar que, durante años se ha pretendido subestimar la presencia de Demodex folliculorum, 1,7 al señalar que es un ácaro saprófito de la piel e inofensivo, la investigación permite sostener que es un parásito frecuente con expresión clínica importante. Por otra parte, todos los pacientes que tienen el ácaro en sus párpados presentan la clínica de blefaritis aunque con variaciones; es claro también que, la clínica depende de la carga parasitaria.

En concordancia con diferentes autores, 7, 9, 13, 25, 27 no debe subestimarse el potencial patógeno de Demodex folliculorum en su vinculación con la blefaritis en humanos o con otras afecciones de la piel, corresponde así, su sospecha ante clínica ocular o dermatológica como la descripta, su reconocimiento y la indicación de tratamiento. La medicación contra el ácaro condujo al rápido alivio de los síntomas o a la mejora de la clínica dermatológica en cuadros de acné o rosácea. Frente al rango variado de síntomas oculares, muchos pacientes toleran la afección por lapsos prolongados y concurren a la consulta en último término. La presencia de Demodex folliculorum de forma asintomática, de acuerdo a nuestra experiencia no es posible, y siempre cursa con clínica; por lo que se debe agudizar la pesquisa para el reconocimiento de varios o de algunos de los síntomas o signos; por ejemplo el prurito ocular, que en el $100 \%$ de los casos de blefaritis por el ácaro estuvo presente. El estudio expone la alta prevalencia de la blefaritis acaricida teniendo en cuenta una muestra importante de consultas oftalmológicas y tiene el propósito de contextualizar estadísticamente la afección. Creemos oportuno poner al corriente sobre una parasitosis frecuente pero subestimada e informar de nuestra experiencia al profesional médico y a la comunidad para motivar la consulta precoz y disminuir los estándares de prevalencia en la población.

\section{Bibliografía}

1. Atias-Neghme: Parasitología Clínica. $3^{\circ}$ Edición. Publicaciones técnicas Mediteráneo. Santiago-Chile. 1992, p. 488-89.

2. Ayres S, Mihan R. Rosacea like Demodicidosis involving the eyelids. Arch Derm 1967; 95: 63-66.

3. Akilov OE, Mumcuoglu KY.Inmune response in demodicosis.J EurAcadDermatolVenereol. 2004, 18: 440-4.

4. Corredor-Osorio, R, Nava Castañeda A, Tovilla Canales J L, Tovilla y Pomar JL, Muñoz Salas, S. Blefaritis por Demodexfolliculorum.Julio-Agosto, 2000.Rev FacMed UNAM Vol.43 (4).

5. Duke-Elder S. Diseases of the eyelids. L Duke Elder S: System of ophthalmology Vol. XIII Part I. Henry Kimpton. London, 1974: 225-230.

6. English FP, Iwamoto T, Darrell RW, DeVoe Agosto. The vector potential of Demo- 
dexfolliculorum. Arch Ophthalmol 1970, 84:83-85.

7. Elston,D. DemodexMites:Facts and controversies. Clinics in Dermatology.2010, 28 (1): 502-504.

8. Gao YY, Di Pascuale MA, Li W, et al. High Prevalence of Demodex in Eyelashes with Cylindrical D-andruff. Invest Opthalmol Vis Sci. 2005; 46(9): 3089-3094.

9. Jarmuda S, O'Reilly N, Zaba R, et al.The potential role of DemodexFolliculorum mites and bacteria in the induction of rosacea.Journal of Medical Microbiology. 2012; 61(11): 1504-1510.

10. Junemann A. Demodexfolliculorum in chronic blepharitis. Online Journal of Ophthalmology.1998. http://www.onjoph.com/english/demodex.html.Accessed May $20,2011$.

11. Kemal M, Sümer Z, Toker MI, Erdoan H, Topalkara A, Akbulut M.The Prevalence of Demodexfolliculorum in blepharitis patients and the normal population.Ophthalmic Epidemiol. 2005 Aug; 12(4): 287-90.

12. Kheirkhah A, Casas V, Li W, Raju V, Tseng S. Corneal manifestations of ocular Demodex infestation. American Journal of Ophthalmology. 2007, 143(5): 743-749.

13. Lacey N, Delaney S, Kavanagh K, Powell FC. Mite-related bacterial antigens stimulate inflammatory cells in rosacea. Br J Dermatol. 2007;157:474-81.

14. Lacey N, Kavanagh K, Tseng SCG.Under the lash Demodex mites in human diseases.Biochem (Lond). 2009; 31(4): 2-6.

15. Liu J, Sheha H, Tseng SCG.Pathogenic role of Demodex mites in blepharitis.CurrOpin Allergy ClinImmunol. 2010; 10(5): 505-510.

16. Lloyd McKernan, A, O’Dwyer V. \& Murphy, O. The Prevalence of DemodexFolliculorum on Eyelashes of Symptomatic and Asymptomatic Normal Patients. Budapest, May 2015. Poster presentation, European Academy of Optometry.

17. Norn MS. Incidence of Demodexfolliculorum on skin of lids and nose.Acta ophthalmologica 1982; 60: 575-583.

18. Del Ponte E. Manual de Entomología Médica y Veterinaria Argentinas. Ediciones Librería del Colegio. Buenos Aires. 1958; p. 265-288.

19. Pena Gil Patrus, Andrade FilhoJosé de Souza. Is demodex really non-pathogenic?. Rev. Inst. Med. trop. S. Paulo.May-June, 2000; 42 (3): 171-173.

20. Pizzi HL, Sánchez RS, Huck GA: Parasitología y Micología Médicas. UNC. 1997. Tomo 3.

21. Pizzi HL, Sánchez RJ, Huck GA. Artrópodos su impacto en la Salud. Ed. Rotagraf. Córdoba.2010, p. 53-54.

22. Pizzi HL, Sánchez RJ, Huck GA, Tomas AF. Artrópodos.Calentamiento global, nuevos desafíos, control de plagas.Ed. Rotagraf. Córdoba. 2012, p. 71-77.

23. Post CF, Juhlin E. Demodexfolliculorum and blepharitis.Arch Dermatol. 1963;88:298-302.

24. Post CF, Juhlin E. Demodexfolliculorum and blepharitis.Arch Dermat 1965; 92 : $542-544$.

25. Rodríguez AE, Ferrer C, Alió JL.Chronic blepharitis and Demodex. Arch SocEspOftalmol, 2005; 80(11):635-42.

26. Roth AM. Demodexfolliculorum in hair follicles of eyelid skin.Annals of Ophthalmology. 1979; 11(1): 37-40.

27. Serrano P. Dermatosis inducidas por Demodexfolliculorum. Piel. 2011; 287: 5. Disponible en: http://www.elsevier.es/sites/default/files/elsevier/eop/S02139251\%2811\%2900190-0. pdf [accedido el 26 de diciembre de 2011].

28. Smith S, McCullen C. Demodexfolliculorumpalpebrum.Canad J Ophthalmol 1969; 4: $3-15$.

29. Türk M, Oztürk I, Sener AG, Küçükbay S, Afar I, Maden A. Comparison of incidence of Demodexfolliculorum on the eyelash follicule in normal people and 
blepharitis patients. TurkiyeParazitolDerg. 2007, 31(4): 296-7.

30. Wesolowska M, Knysz B, Reich A, Blazejewska D, Czarnecki M, Gladysz A, Posowski A, Misiuk-Hojlo, M. Prevelence of Demodex spp.in eyelash follicles in different populations. Archives of Medical Science.2014, 10(2), 319-324.

31. Urbina F, Plaza C, Posada C. Actas Dermosifiliogr.2003; 94 (2):119-20.

32. Zhao Y, Wu LP, Hu L, Xu JR. Association of blepharitis with Demodex: a meta-analysis. Ophthalmic Epidemiology.2012; 19(2), 95-102. 